\title{
Method of systematic determination of specific thermal characteristics of building
}

\author{
Vitaliy Prokhorov ${ }^{1, a}$ \\ ${ }^{1}$ Moscow State University of Civil Engineering, Russia
}

\begin{abstract}
In the paper the classical term «specific thermal characteristics of building» proposed by professor Chaplin V.M. for heating systems developing also for others heat-consuming system for building such as ventilation, air conditioning, hot-water supply system is observed. The followings attributing values are established: external building volume, temperature potential in annual crevice cycle.
\end{abstract}

\section{Introduction}

The term «specific thermal characteristics of building» was proposed by prof. Chaplin M.V. more than 100 years ago. Primarily this characteristics was applied for pre-project evaluation of followings parameters:

a) capacity of heating systems and equipment;

b) tentative fuel requirement of building;

c) sufficiency and optimality of thermal protection of buildings.

This characteristic are widely used at present time including educational programs because the specific capacity of heating systems per $1 \mathrm{~m} 3$ of building measured by external dimension. The new application fields of this parameter are proposed for normalization of building energy consumption reflected its energy efficiency.

Taking into account the modern building has not only heating system but also three types of thermal-consuming systems (mechanical ventilation, air conditioning, hot-water supply system) that are not completely described in regulatory documents the technical reasonability of extending of the term «specific thermal characteristics of building» for each heating system type takes place. It allows correct estimation of annual total energyconsuming in the building.

This idea and concept of the calculation procedures are proposed in 2002 [1] in form of equations.

Further development of this idea is reflected in research works [2, 3 etc.], regulatory documents $[4,5]$, works of developers of the document «SP50.13330-2012» [6] as well as the principle paper about «boundary of analysis» [7], required addition and refinement of earlier data.

The paper is devoted to realization of calculation method and the above concepts when design process.

\footnotetext{
${ }^{a}$ Corresponding author: ProkhorovVI@mgsu.ru
} 
The necessary requirements are followings:

1) application for every type of heat consumption systems using external building volume Vext for calculation of all rooms [7];

2) justification of temperature differential for each engineering system (potential heat exchange method by V.N. Bogoslovsky) for every design condition.

Heating systems

In this work the classical form of parameter «specific thermal characteristic of building» is following:

$$
q_{\text {heat }}^{c}=\frac{Q_{\text {heat }}^{C B}}{V_{\text {ext }} \cdot\left(t_{\text {int }}-t_{\text {ext }}\right)}, \mathrm{W} / \mathrm{m}^{3}{ }^{\circ} \mathrm{C}
$$

where: $q_{\text {heat }}^{c}$ is calculated specific thermal characteristic of building, applying for heating systems;

$Q_{\text {heat }}^{c b}$ calculated balanced building heat losses, W.

$V_{\text {ext }}$ is external building volume including all envelopes having a heat exchange with the environment (ventilated facades, heated underground floors, glazed balconies), $\mathrm{m}^{3}$.

$t_{\text {int }}$ is computation average internal air temperature of building, ${ }^{\circ} \mathrm{C}$.

$t_{\text {ext }}$ is computation external temperature for climate zone of construction, ${ }^{\circ} \mathrm{C}$.

Determination of parameters $q_{\text {heat }}^{c}$ and $Q_{\text {heat }}^{c b}$ taking into account transmission heat losses and heat load on natural ventilation: controlled and uncontrolled (infiltration and exfiltration) is the basic procedure when design of energy balance of building in case of heating system.

The main idea of equation (1) is ratio of capacity of heating system $Q_{\text {heat }}^{c b}$ to calculated value (tint -text) in working medium (generally, it is air) for internal and external side of building envelope per unit of heat exchange potential. Ratio of heat consumption value (per $1 \mathrm{oC})$ or «specific» capacity to $V_{\text {ext }}$ is specific thermal characteristics of building in case of heating system.

Next stage is to determine analogue values for others heat consuming systems of building by calculation method. Realization of this stage demonstrates comparable (or higher) values of analogues vs. value of $q_{\text {heat }}^{c}$. Thus, they can be used according to common recommendations of energy performance certificate, particularly, for determination of annual heat consumption.

So, extending of term «specific thermal characteristic of building» consists in individual application not to heating system only but for others mechanocaloric systems of building that follows in the Chaplin V.M. theory.

It should be noted, in the bellow methods the common denominator in «external task» (heat-mass exchange of building with environment medium according to prof. V.P. Titov) must be used for constant value of external building volume $V_{\text {ext }}$ according to eq. (1), but not internal building volume served by certain mechanocaloric system only. These methods are suitable for «internal tasks» (according to prof. V.P. Titov) only, for exp. when design of air-heat microclimate system as well as when comparison of performance indicator (for exp., energy consumption) for certain systems and served buildings. 
Calculated value of (tint -text) should be in compliance with heat conductor and operating conditions of certain system.

More detail description of methods is presented bellow.

Systems of mechanical ventilation

$$
q_{\text {vent }}^{c}=\frac{Q_{\text {vent }}^{c}}{V_{\text {ext }}\left(t_{\text {in }}-t_{\text {ext }}\right)}, \mathrm{W} / \mathrm{m}^{3} \mathrm{C} \mathrm{C}
$$

$Q_{\text {vent }}^{c}$ thermal capacity of systems with mechanical ventilation in building, (W);

$V_{\text {ext }}$ - the building volume, m3; ${ }^{t_{\text {in }}}$ - calculate temperature of inlet air, ${ }^{\circ} \mathrm{C} ;{ }{ }_{\text {ext }}$ - calculate temperature of external air, $\mathrm{oC}$ for ventilation systems during winter season.

During a summer season the ventilation systems work without air heating, i.e. only electric energy consumption takes place.

Air conditioning system

Heat consumption of conditioner depends of applied air handling scheme. In this work the simplified system (straight-through arrangement) is studied.

As air heating in central air-conditioner is accomplished annually («reheat») the calculation of «specific thermal characteristic» value for building is accomplished for winter and summer seasons separately.

Winter season

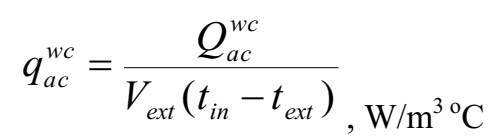

where $Q_{a c}^{w c}$ thermal capacity of air heating during winter season («preheat» and «reheat» if it takes place) taking into account calculated parameters, $\mathrm{W}$;

$t_{\text {in }}$ - temperature of inlet air, ${ }^{\circ} \mathrm{C}$.

During winter season the air heating process with aspirator in many cases (exp. in air conditioners for textile industry) is not taken account. In others cases the difference between $t_{\text {in }}$ and $t_{\text {ext }}\left(t_{i n}-t_{\text {ext }}\right)$ is reduced. Required capacity for «reheat» effect is described with the below scheme for summer season.

Summer season

To provide the temperature of inlet air equal to heat-to-humidity ratio in the building for achievement a required humidity after cooling and drying process the air should be heated even during a summer season (Fig. 1). 


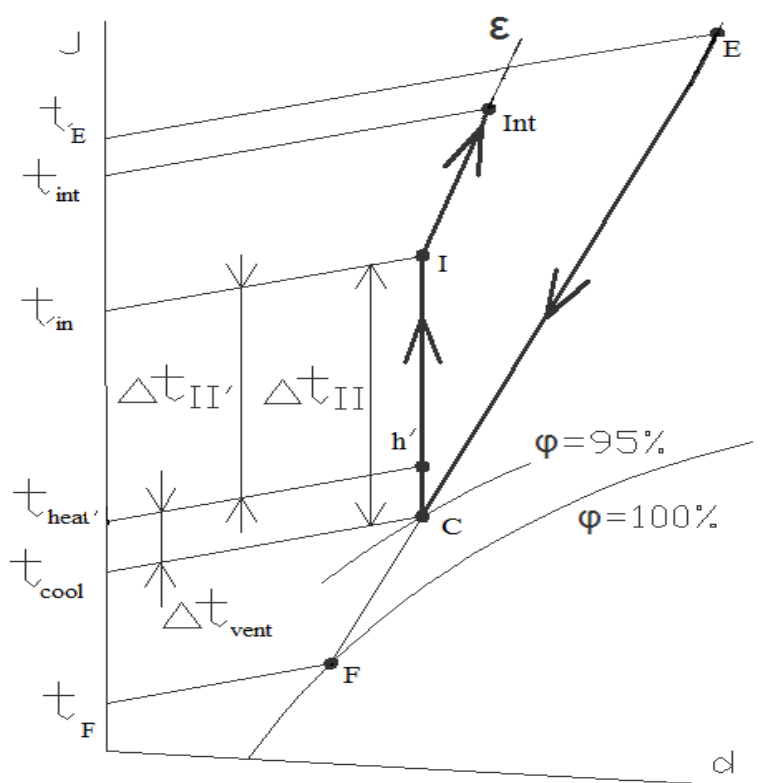

Fig. 1. Explanatory scheme for heat consuming processes during a summer season. $\Delta$ tvent $=($ theat $-\mathrm{tc})-$ potential air heating with ventilator;

$\varepsilon$ is heat-to-humidity ratio in room;

$\Delta t_{I I^{\prime}}=\left(t_{\text {in }}-t_{\text {heat }}\right)$ is air heating with heating element («reheat») taking into account its partial heating with ventilator;

$\mathrm{I}-\mathrm{C}$ - internal process;

E-C-F - cooling and drying of external air

$$
q_{a c}^{s c}=\frac{Q_{a c}^{s c}}{V_{e x t}\left(t_{i n}-t_{c o o l}\right)}, \mathrm{W} / \mathrm{m}^{3 \mathrm{o}} \mathrm{C}
$$

where: $Q_{a c}^{s c}$ calculated thermal capacity of air heating («reheat») for summer season, W;

$t_{\text {in }}$ - temperature of inlet air, oC;

$t_{\text {cool }}$ - temperature of air after cooling, oC.

If in this scheme a ventilator is placed after air-cooling system the value of $\Delta t_{\text {vent }}$ (difference between tc and theat) should be taken account (see Fig. 1).

To simplify the equations, hereinafter the parameter tc is used only (i.e. $\Delta t_{\text {vent }}=0$ ).

Also, the ratio of mass flows for both interacting mediums should be considered as air heating is realized in heat exchanger with water. So, the value $Q_{a c}^{s c}$ can be determined for air and water.

Balance equation of heat-exchange is following:

$$
Q_{a c}^{s c}=G_{i n} \cdot c_{\text {air }} \cdot\left(t_{\text {in }}-t_{c}\right)=G_{\text {in }} \cdot c_{\text {air }} \cdot \Delta t_{I I}=G_{w} \cdot c_{w} \cdot \Delta t_{w}
$$

where: Gin and Gw are mass flow of heated inlet air and heating water in air heater, $\mathrm{kg} / \mathrm{s}$; $\mathrm{c}_{\text {air }}$ and $\mathrm{c}_{\mathrm{w}}$ are heat capacities for air and water; 
$\Delta \mathrm{t}_{\mathrm{w}}$ is temperature difference between input and output of heated water in heat exchanger.

Return temperature of water in heat exchanger depends of initial temperature of interacting flows, air and water flow rates in obstruction-free flow areas and obtained by traditional calculation method. Input water temperature during a summer season is equal to temperature in hot water supply system.

In scheme where water in «reheat» conditioner after water heat exchanger is used for hot water supply system, its temperature can be equal to 55 oC according to SP30-133302012 for water supply system in building (i.e. water temperature in water tap).

Advantage of this scheme is highly-accurate monitoring in case of low heat load and small temperature differences in air flow that typical for «retreat» effect. In case of «retreat» effect, air-heater in conditioner is directly connected with flow line on heat system, the max temperature during summer season is $70{ }^{\circ} \mathrm{C}$ (during winter season this value is higher that requires reconnection of heat exchanger to return line of heating system).

Temperature differences in these cases presented a heat-change potential for water are followings: $\Delta t_{w}=55-\mathrm{t}_{\text {wh }}^{s}$ and $\Delta t_{w}=70-\mathrm{t}_{\text {wh }}^{r}$ where $\Delta t_{w h}^{\pi}$-average temperature of return temperature of water calculated for «reheat» air heater.

Temperature differences of air and water flows in «reheat» heat exchanger are related in followings equations:

$$
\begin{aligned}
& \Delta t_{I I}=\Delta t_{w} \frac{G_{w} \mathrm{c}_{\mathrm{w}}}{G_{i n} \mathrm{c}_{\mathrm{air}}} \\
& \Delta t_{w}=\Delta t_{I I} \frac{G_{i n} \mathrm{c}_{\mathrm{air}}}{G_{w} \mathrm{c}_{\mathrm{w}}}
\end{aligned}
$$

According to eq. (6) and (6') as well as at $t_{w}=55$ the value of specific thermal capacity can be shown by following:

$$
\begin{gathered}
\frac{Q_{a c}^{s c}}{t_{\text {in }}-t_{\text {heat }}}=\frac{Q_{a c}^{s c}}{55-t_{w h}^{s}} \cdot \frac{G_{i n}}{G_{w}} \cdot \frac{1}{4.19} \\
\frac{Q_{a c}^{s c}}{55-t_{w h}^{s}}=\frac{Q_{a c}^{s c}}{t_{\text {in }}-t_{\text {heat }}} \cdot \frac{G_{w}}{G_{\text {in }}} \cdot \frac{4.19}{1}
\end{gathered}
$$

Hot-water supply system

For these systems the determination of specific thermal characteristics of building is differed for summer and winter seasons due to variety of cold water temperature.

Winter season

$$
q_{h w}^{w c}=\frac{Q_{h w}^{w c}}{V_{e x t}\left(55-t_{c w}^{w c}\right)}, \mathrm{W} / \mathrm{m}^{3{ }^{\circ} \mathrm{C}}
$$

where $Q_{h w}^{w c}$ - calculated thermal capacity of hot-water supply system for winter season, W; $t_{h w}^{w c}$ - computation temperature of cold (tap) water during a winter season, ${ }^{\circ} \mathrm{C}$ (for exp. $5-8{ }^{\circ} \mathrm{C}$ ).

Summer season

$$
q_{h w}^{s c}=\frac{Q_{h w}^{s c}}{V_{e x t}\left(55-t_{c w}^{s c}\right)}, \mathrm{W} / \mathrm{m}^{3{ }^{\circ} \mathrm{C}}
$$


where: $Q_{h w}^{s c}$ - calculated thermal capacity of hot-water supply system for summer season, $\mathrm{W}$;

$t_{c w}^{s c}$ - computation temperature of cold (tap) water during a summer season, ${ }^{\circ} \mathrm{C}$ (for exp. $\left.12-15^{\circ} \mathrm{C}\right)$.

Note, the values $Q_{h w}^{w c}$ and $Q_{h w}^{s c}$ include heat losses through pipes due and to daily and seasonal irregularity of hot water consumption.

As the denominator in the eq. (8) and (9) for specific thermal characteristics of building is the temperature difference of water vs. others heat consuming systems where temperature difference of air is used, the reasonability of introduction of universal analog parameter for air heating process with the same thermal capacity with using eq. (6) and (7) takes place.

Data obtained with calculation methods demonstrate the application of mathematical analysis is not effective because the values of $Q_{h w}^{w c}$ and $Q_{h w}^{s c}$ in the eq. (8) and (9) are equal as well as value of temperature differences for air and water (heat exchange potential) in real heat exchanger are varied not significantly (in case of subregeneration) or are equal (in case of ideal heat exchange process).

\section{Conclusion}

Mass air and water flows correlate with their heat capacities and are used when $Q_{h w}^{w c}$ and $Q_{h w}^{s c}$ determination.

Mathematical form for winter season is following:

$$
q_{h w}^{s c}=\frac{Q_{h w}^{s c}}{V_{\text {ext }}\left(55-t_{c w}^{s c}\right)}=\frac{Q_{h w}^{s c} G_{h w}}{V_{\text {ext }}\left(t_{\text {final }}-t_{\text {initial }}\right) G_{c o n d}} \cdot \frac{4.19}{1}
$$

where: $G_{h w}$ - mass hot water flow, $\mathrm{kg} / \mathrm{s}$;

$G_{\text {cond }}$ - mass conditional air flow, $\mathrm{kg} / \mathrm{s}$ when air heating from calculated external air temperature for winter season $t_{\text {initial }}$ to final temperature $t_{\text {final }}$ for heating system.

Value of this temperature can be determined by using statistical value of subregeneration for real air-water heat exchanger and following determination of nominal air flow Gcond using the following parameters: standard hot water flow $G_{h w}, t_{c w}^{w c}, \mathbf{t}_{\mathrm{ext}}$ and heat capacities of medium:

$$
Q_{h w}^{s c}=G_{h w} \cdot 4.19\left(55-t_{c w}^{w c}\right)=G_{c o n d} \cdot 1\left(t_{f-c o n d}-t_{\mathrm{ext}}\right)
$$

Analogous equations with parameters of $t_{c w}^{s c}, t_{\text {initial }}^{s}, t_{f \text {-cond }}$ are can be applied for determination of $G_{\text {cond }}$ for summer season.

$$
G_{c o n d} q_{h w}^{s c}=\frac{G_{h w} \cdot 4,19\left(55-t_{c w}^{s c}\right)}{\left(t_{f-c o n d}^{s c}-t_{\text {initial }}^{s}\right)}
$$

\section{References}

1. V.I. Prokhorov, Stroitelni Expert 13(16), 127-131 (2002) 
2. V.I. Prokhorov, Advanced systems of heat and gas supply and ventilation (2003)

3. V.I. Prokhorov, House construction 1 (2012)

4. Organizations standard STO17532043-001-2005 (2006)

5. SP 50.13330.2012 (2012)

6. V.G. Gagarin, V.V. Kozlov, Association of HVAC Engineers. Northwest 1 (2012)

7. V.I. Prokhorov, Web-Bulletin VolgGASU. Multidisciplinary series 2 (2014)

8. Yu.P. Grigoriev, Stroitelni Expert 1 (2003) 